\title{
Organic Photovoltaics
}

\section{Tackling Voltage Losses in OPVs}

\section{A powerful theoretical model encompassing hybridization and thermal population of vibronic states was developed to explain the reduced non-radiative voltage losses in highly efficient non-fullerene acceptor organic solar cells.}

Natalie Banerji

For decades, organic photovoltaics (OPVs) were dominated by blends of conjugated polymer donors with electronaccepting fullerene derivatives. ${ }^{1}$ A paradigm shift has occurred since 2015 , with the introduction of efficient small molecule non-fullerene acceptors (NFAs). ${ }^{2}$ Their superior performance has led to record OPV efficiencies of over $18 \%,{ }^{3}$ re-launching organic solar cells to the forefront of attention. Unlike fullerenes, NFAs show high absorption in the visible and near-infrared region, which can be complemented with the donor polymer to cover a broad spectral range for light harvesting. Moreover, charge transfer at the polymer:NFA interface is efficient even with a very low or absent driving force, ${ }^{4}$ which improves the open circuit voltage $\left(\mathrm{V}_{\mathrm{OC}}\right)$ due to the near-zero offset between the locally excited (LE) state of the low-bandgap component and the interfacial charge transfer (CT) state. ${ }^{5}$ Nevertheless, voltage losses remain the main limitation of organic solar cells with respect to hybrid and inorganic technologies, and in particular non-radiative voltage losses $\left(\Delta \mathrm{V}_{\mathrm{nr}}\right)$ need to be better understood and further minimized. Providing essential design rules in this direction, Chen et al. have closely examined $\Delta \mathrm{V}_{\mathrm{nr}}$ for a large number of fullerene- as well as NFA-based systems, presenting their theoretical and experimental results in Nature Energy. ${ }^{6}$ Taking into account hybridization effects between the LE and CT states as well as the thermal population of those states, they arrive at a unified description of voltage losses in organic solar cells.

For conventional OPVs containing fullerene acceptors, it has been shown that both the $\mathrm{V}_{\mathrm{OC}}$ and $\Delta \mathrm{V}_{\mathrm{nr}}$ scale with the energy of the CT state. ${ }^{7,8}$ This can be explained by the energy gap law, which stipulates that the rate of nonradiative transitions in the weak coupling regime increases exponentially with a decreasing energy difference between the initial and final states. It is therefore expected that non-radiative recombination from the CT to the ground state (G), which is at the origin of $\Delta V_{n r}$, is strongly enhanced for OPV systems with low-lying CT states. As optimized NFA blends with absorption reaching far into the near-infrared range have their CT states at low energy, they should in principle perform poorly in terms of voltages losses. However, contrary to expectations, reduced $\Delta \mathrm{V}_{\mathrm{nr}}$ is observed in state-of-the-art NFA devices, contributing to their record performance. Chen et al. use a vibronic three-state model to rationalize this observation (Figure 1A). This takes into account hybridization (i.e. the formation of mixed states due to electronic coupling between the LE and CT states at small energetic offset) as well the thermal population of the LE and CT states when calculating the radiative and non-radiative recombination rates.

The authors start by simulating the emission spectra for different degrees of hybridization using the three-state model and compare this to experimental electroluminescence (EL) data recorded at different temperatures. In EL measurements, emission results predominantly from the recombination of injected carriers at the D:A interface and is therefore selective to interfacial states. The experimental EL spectra for a variety of D:A systems, having different energy offsets $\left(\Delta \mathrm{E}_{\mathrm{LE}-\mathrm{CT}}\right)$ and electronic couplings ( $\mathrm{L}_{\mathrm{LE}-\mathrm{CT}}$ ), could be well reproduced with the model, allowing to extract the relevant parameters from the spectroscopic data. At low temperature $(30 \mathrm{~K})$, the emission is dominated by the CT state and becomes more intense as $t_{L E-C T}$ is increased, due to intensity-borrowing from the LE state. At room temperature, the emission is similar to that of the LE state at weak $t_{L E-C T}$, while it contains LE and CT contributions at intermediate coupling and is dominated by the CT state for important hybridization. The effect seen 
with small $t_{\mathrm{LE}-\mathrm{CT}}$ is due to thermal population of the LE state and depends strongly on having a small $\Delta \mathrm{E}_{\mathrm{LE}-\mathrm{CT}}$ so that the vibronic LE states become thermally accessible.
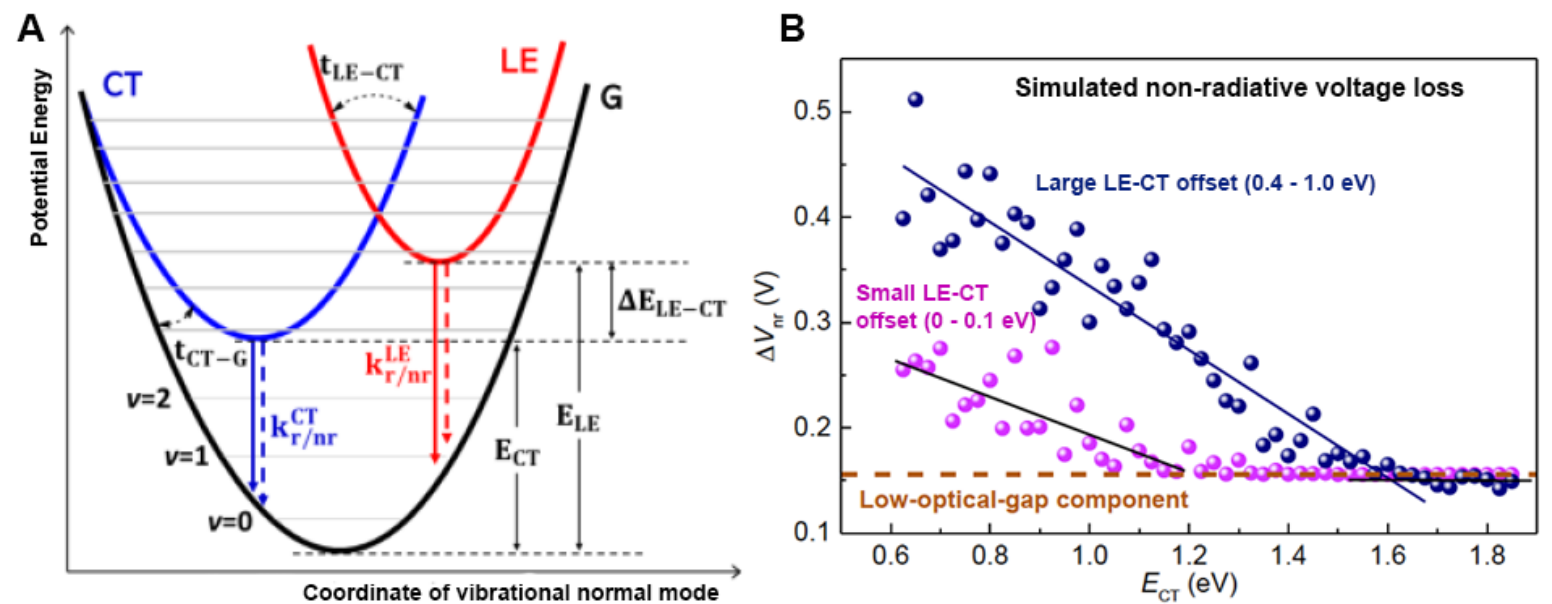

Figure 1. Vibronic three-state model and simulation of the non-radiative voltage losses. A) Schematic representation of the potential energy curves used in the three-state vibronic model, showing the ground state $(G)$, the locally excited state (LE) and the charge transfer state (CT). $E_{\mathrm{CT}}$ and $\mathrm{E}_{\mathrm{LE}}$ are the energies of the states and $\Delta \mathrm{E}_{\mathrm{LE}-\mathrm{CT}}$ is their energetic offset. The $\mathrm{t}_{\mathrm{CT}-\mathrm{G}}$ and $\mathrm{t}_{\mathrm{LE}-\mathrm{CT}}$ represent the electronic coupling of the CT state with the $\mathrm{G}$ state and LE state, respectively, while the blue and red lines show the radiative and nonradiative decay rates. The horizontal gray lines are the vibronic levels of the hybrid state. $B)$ Simulated non-radiative voltage loss $\left(\Delta V_{n r}\right)$ as a function of $\mathrm{E}_{\mathrm{CT}}$, with $\Delta \mathrm{E}_{\mathrm{LE}-\mathrm{CT}}$ randomly distributed in the range $0.4-1.0 \mathrm{eV}$ or $0-0.1 \mathrm{eV}$, and $\mathrm{t}_{\mathrm{LE}-\mathrm{CT}}$ randomly distributed between 1-50 meV.

In the next stage, the effect of $\Delta \mathrm{E}_{\mathrm{LE}-\mathrm{CT}}$ and $\mathrm{t}_{\mathrm{LE}-\mathrm{CT}}$ was related to the radiative and non-radiative voltage losses by simulating their impact on the decay rate constants of the LE and CT states. For large offsets (>0.3 eV), such as are common in fullerene-based systems, the radiative decay rate of the CT state is increased by even weak hybridization with the much more emissive LE state, but the non-radiative decay is hardly affected. In this regime, $\Delta \mathrm{V}_{\mathrm{nr}}$ scales linearly with $\Delta \mathrm{E}_{\mathrm{LE}-\mathrm{CT}}$ and the $\mathrm{CT}$ energy $\left(\mathrm{E}_{\mathrm{CT}}\right)$ according to the three-state model (Figure 1B), in agreement with the predictions from energy gap law and with experimental observations (that the authors have assembled for a large collection of reported blends). Things become unexpected when $\Delta \mathrm{E}_{\mathrm{LE}-\mathrm{CT}}$ decreases to small values $(<0.3 \mathrm{eV})$, which is the case in efficient NFA-based systems. Now the thermal population of the LE state plays the predominant role (irrespective of hybridization) and determines the radiative and non-radiative decay rates. In this case, $\Delta \mathrm{V}_{\mathrm{nr}}$ no longer scales with $\mathrm{E}_{\mathrm{CT}}$ (i.e. no longer obeys the energy gap law) and undergoes an important reduction, approaching the value of the low-bandgap component (Figure 1B). Chen et al. confirm those theoretical predictions with experimental data obtained for different NFA materials. These data cannot be rationalized with a simple two-state model that does not take into account state hybridization and thermal populations. To minimize the non-radiative voltage loss for low offset blends, a high emission yield of the LE state rather than of the CT state is required. Therefore, fullerene systems with low offsets do not show reduced $\Delta \mathrm{V}_{\mathrm{nr}}$, since the LE state is only weakly emissive. Finally, it is shown for a broad range of systems that efficient charge generation can occur even for low offset systems and therefore does not require a trade-off with the minimization of voltage losses.

The impact of the work presented by Chen et al. resides in the powerful theoretical model, which does not only take into account hybridization between the LE and CT states, but also their thermal population. This provides a 
generalized description of voltage losses in OPV blends, valid for conventional fullerene-based systems as well as for novel highly efficient NFA-based materials. With this model, the experimental EL emission spectra as well as the voltage losses can be correctly predicted. Microscopic parameters such as the extent of LE-CT hybridization can thus be extracted from simple spectroscopic data. The model explains deviations from the energy gap law that are observed for efficient NFA-based OPV systems with low energetic offsets for charge transfer. Encouraging design rules emerge, that show that there is still great potential to improve the efficiency of OPVs by targeting blends with low energetic offsets, complementary absorption that extends far into the near-infrared range and a low-bandgap component with high emission quantum yield. The findings published in Nature Energy are a significant step forward in overcoming the main challenge of organic solar cells, which is the significant voltage loss. This holds promise of competitive performance with inorganic and hybrid technologies, coupled to other advantageous properties of OPVs, such as light-weight, flexibility, low processing cost and non-toxicity.

\section{References}

1. Thompson BC, Fréchet JMJ. Polymer-fullerene composite solar cells. Angew. Chem., Int Ed Eng/ 2008, 47(1): 58-77.

2. Hou J, Inganas O, Friend RH, Gao F. Organic solar cells based on non-fullerene acceptors. Nat. Mater. 2018, 17(2): 119-128.

3. Zhang M, Zhu L, Zhou G, Hao T, Qiu C, Zhao Z, et al. Single-layered organic photovoltaics with double cascading charge transport pathways: $18 \%$ efficiencies. Nat. Commun. 2021, 12(1): 309.

4. Zhong Y, Causa' M, Moore GJ, Krauspe P, Xiao B, Günther F, et al. Sub-picosecond charge-transfer at near-zero driving force in polymer:Non-fullerene acceptor blends and bilayers. Nat. Commun. 2020, 11(1): 833.

5. Qian D, Zheng Z, Yao H, Tress W, Hopper TR, Chen S, et al. Design rules for minimizing voltage losses in high-efficiency organic solar cells. Nat. Mater. 2018, 17(8): 703-709.

6. Chen X-K, Qian D, Wang Y, Kirchartz T, Tress W, Yao H, et al. A unified description of non-radiative voltage losses in organic solar cells. Nat. Energy 2021.

7. Vandewal K, Tvingstedt K, Gadisa A, Inganäs O, Manca JV. Relating the open-circuit voltage to interface molecular properties of donor:Acceptor bulk heterojunction solar cells. Phys. Rev. B 2010, 81(12): 125204.

8. Benduhn J, Tvingstedt $\mathrm{K}$, Piersimoni F, Ullbrich S, Fan $\mathrm{YL}$, Tropiano $\mathrm{M}$, et al. Intrinsic non-radiative voltage losses in fullerene-based organic solar cells. Nat. Energy 2017, 2(6).

\section{Competing Interest}

The author declares no financial or non-financial competing interest.

\section{Affiliation}

Natalie Banerji; University of Bern; Department of Chemistry, Biochemistry and Pharmacy; Freiestrasse 3, $\mathrm{CH}-$ 3012 Bern, Switzerland. natalie.banerji@dcb.unibe.ch 